\title{
Toxoplasma gondii: Manipulation of host cell machinery in the journey from intestine to brain
}

\author{
Wan Aliaa Wan Sulaiman ${ }^{\mathrm{a}, \mathrm{d}, *}$, Mohd Hazmi Mohamed ${ }^{\mathrm{b}}$, Ramachandran Vasudevan ${ }^{\mathrm{d}}$, \\ Siew Mooi Ching ${ }^{\mathrm{c}, \mathrm{d}}$, Sazlyna Mohd Sazlly Lim ${ }^{\mathrm{a}, \mathrm{d}}$, Liyana Najwa Inche Mat ${ }^{\mathrm{a}, \mathrm{d}}$, \\ Fan Kee Hoo ${ }^{\mathrm{a}, \mathrm{d}}$, Hasnur Zaman Hashim ${ }^{\mathrm{a}}$, Wan Zulhaikal Hafiz Wan Zukiman ${ }^{\mathrm{a}}$, \\ Hamidon Basri ${ }^{\mathrm{a}}$ \\ a Department of Medicine, Faculty of Medicine and Health Sciences, Universiti Putra Malaysia, Serdang, Malaysia \\ b Department of Surgery, Faculty of Medicine and Health Sciences, Universiti Putra Malaysia, Serdang, Malaysia \\ ${ }^{\mathrm{c}}$ Department of Family Medicine, Faculty of Medicine and Health Sciences, Universiti Putra Malaysia, Serdang, Malaysia \\ d Institute of Gerontology, Universiti Putra Malaysia, 43400, Serdang, Selangor, Malaysia
}

\section{A R T I C L E I N F O}

\section{Article history:}

Received 24 January 2017

Accepted 13 April 2017

Available online 22 April 2017

\section{Keywords:}

Toxoplasmosis

Host-pathogen interactions

Central nervous system diseases

\begin{abstract}
A B S T R A C T
Question: From previous work, this group had discovered that infection of dendritic cells (DC) caused by the intracellular parasite (Toxoplasma gondii) induced a hyper-migratory state in DC which was a possible mechanism of the protozoan dissemination. For this study, their question was whether $\Upsilon$-aminobutyric acid (GABA) signaling was the possible mechanism underlying the control to the migratory properties of the infected DC.

Methods: To affirm the hypothesis, a series of complimentary experiments were conducted using both animal and human DC, also in vitro and in vivo.

Main results: The main results of the experiments were as follows:

First, murine and human DC produced GABA upon $T$. gondii infection. By quantitatively measuring GABA in the supernatant by enzyme-linked immunosorbent assay (ELISA), they found that live Toxoplasma infection of mouse DC, from the three predominant genotypes, increased GABA and the secretion occurred rapidly following infection. In addition, the fluorescence-activated cell sorting (FACS) of infected DC populations demonstrated that GABA production happened mainly in green fluorescent protein (GFP) + DC.

Second, functional GABA receptors were expressed in mouse and human $D C$. The $G_{A B A}$ receptors of a3, $ß 3$, and $\mathrm{r} 1$ subunit expression was determined with quantitative reverse transcription polymerase reaction (RT-qPCR). Subsequent immune-cytochemical stainings indicated expression of the $\beta 3$ subunit in both infected and non infected DC. These receptors were proved to be functional in the electrophysiology tests as membrane currents were induced in $T$. gondii-infected DC.

Third, transmigration of infected DC in vitro was reduced by targeting GABA synthesis and transport. Addition of inhibitors of GABA system such as glutamic acid decarboxylase (GAD) inhibitor (SC) and gamma-aminobutyric acid transporter 4 (GAT4) inhibitor (SNAP) to the infected DC had significantly decreased the secretion of GABA and also the transmigration of infected DC.

Fourth, the motility and chemotaxis of infected DC in vitro was modulated by GABAergic signaling in vitro. In a chemotaxis chamber system which consisted of chemokine CCL19 concentration gradient, Toxoplasma-infected DC demonstrated an increased random directional motility and velocity even in absence of chemokine compared to non-infected DC.

Finally, up-regulation of CCR7 in human and mouse DC were observed upon T. gondii infection in vitro. Furthermore, the dissemination of $T$. gondii in vivo was reduced by GABAergic inhibition in adoptively transferred infected DC as shown in the bioluminescence imaging (BLI) studies. Photonic emissions were also observed ex vivo in different organs, and the authors noted significant differences in the emissions on days 1-2 in the brain parenchyma.
\end{abstract}

\footnotetext{
* Corresponding author at: Department of Medicine, Faculty of Medicine and Health Sciences, Universiti Putra Malaysia, 43400, Serdang, Malaysia. E-mail addresses: wanaliaa@upm.edu.my, drwanaliaa@gmail.com (W.A. Wan Sulaiman).
} 
Conclusion: The authors concluded that GABAergic signaling modulated the transmigration of DC and that the intracellular pathogen Toxoplasma gondii seizes the GABAergic signaling of DC like a trojan horse mechanism to assure dissemination.

(c) 2017 INDIACLEN. Published by Elsevier, a division of RELX India, Pvt. Ltd. All rights reserved.

\section{Commentary}

This paper unveils a novel knowledge in the elusive area of mechanism of spread of $T$. gondii by providing the first evidence that GABAergic signaling controls the transmigration of DC and that T. gondii has been taken over this pathway for dissemination. ${ }^{1}$ This new knowledge will certainly incite discussion among the scientific committee since GABA is the principal inhibitory neurotransmitter in the brain, ${ }^{2,3}$ though much more studies need to be done to further verify and explore the findings.

In general, the group's manuscript was well written. A concise introduction was provided, with emphasis on the clinical and pathophysiology of toxoplasmosis, in particular, the fundamentals of it's propagation in hosts. The authors had build on their previous work that identified hyper-migratory properties of DC. ${ }^{4}$ Clear definition of their study hypothesis was given.

The methodology used for their experiments, for example using in vitro models and in vivo BLI in a murine model of toxoplasmosis are practical, complimentary and up-to-date. Furthermore, the methodology described in detailed, enabling future replication of the experiments. Most of the experiments were repeated at least 3 times with complementary experiments were described in the supplementary sections, reinforcing the validity of observed results. Appropriate statistical analyses were performed as they mostly utilised non-parametric model which was relevant to the small sample size.

The results of the study had answered the study's questions, and they were clearly depicted with well-annotated figures and tables. The authors demonstrated that the infected DC secreted GABA and also expressed GABA receptors with the ability to synthesize and transport GABA. Furthermore, the migratory properties of the dendritic cell diminished when GABA function was inhibited. In the discussion, the authors provided a structured presentation and were analytical about interpreting the result. They had also discussed the increasingly recognised concept of 'inflammatory reflex' which was the interaction and influence of neurotransmitters on the immune cell function., ${ }^{2,3}$ Overall, the study results are original and scientifically sound, adding a research value in understanding the unknown mechanism of the $T$. gondii's dissemination through the infected host. ${ }^{5}$

\section{Disclosure of conflict of interest}

The authors have none to declare.

I certify that all my affiliations with or financial involvement in, within the past 5 years and foreseeable future, any organization or entity with a financial interest in or financial conflict with the subject matter or materials discussed in the manuscript are completely disclosed.

\section{Acknowledgement}

The authors would like to thank UPM research fund (grant no: GP-IPM/2014/9427500) for the financial support. The URL is http:// www.rmc.upm.edu.my/.

\section{Appendix A}

The title and complete citation of the original article being discussed in this journal club:

GABAergic Signaling Is Linked to a Hypermigratory Phenotype in Dendritic Cells Infected by Toxoplasma gondii.

Citation: Fuks JM, Arrighi RBG, Weidner JM, Kumar Mendu S, Jin Z, Wallin RPA, et al. (2012) GABAergic Signaling Is Linked to a Hypermigratory Phenotype in Dendritic Cells Infected by Toxoplasma gondii. PLoS Pathog 8(12): e1003051. http://dx.doi.org/ 10.1371/journal.ppat.1003051.

Correspondence address of the discussed paper: Center for Infectious Medicine, Department of Medicine, Karolinska Institutet, Karolinska University Hospital Huddinge, Stockholm, Sweden, 2 Swedish Institute for Communicable Disease Control, Stockholm, Sweden.

Funding of the discussed paper: The study was supported by grants from the Swedish Medical Research Council (to Antonio Barragan and Brindys Birnir; URL http://vrproj.vr.se/default.asp? funk=s), the Swedish Foundation for Strategic Research and a grant from Uppsala University. Jessica M Weidner is the recipent of a postdoctoral fellowship from the Wenner Gren Foundation. Zhe Jin holds a postdoctoral stipend from the Swedish Society for Medical Research. The funders had no role in study design, data collection, and analysis, decision to publish, or preparation of the manuscript.

Link of the discussed paper: http://dx.doi.org/10.1371/journal. ppat.1003051.

http://journals.plos.org/plospathogens/article?id=10.1371/ journal.ppat.1003051\#abstract0.

\section{Reference:}

1. Fuks JM, Arrighi RBG, Weidner JM, et al. GABAergic signaling is linked to a hypermigratory phenotype in dendritic cells infected by Toxoplasma gondii. PLoS Pathog. 2012;8(12).

2. Ben-Ari Y, Gaiarsa J-L, Tyzio R, Khazipov R. GABA: a pioneer transmitter that excites immature neurons and generates primitive oscillations. Physiol Rev. 2007;87(October (4)):1215-1284.

3. Khakhalin AS. Questioning the depolarizing effects of GABA during early brain development. I Neurophysiol. 2011:106(3).

4. Dellacasa-Lindberg I, Fuks JM, Arrighi RBG, et al. Migratory activation of primary cortical microglia upon infection with Toxoplasma gondii. Infect Immun. 2011;79(8):3046-3052.

5. Harker KS, Ueno N, Lodoen MB. Toxoplasma gondii dissemination: a parasite's journey through the infected host. Parasite Immunol. 2015;37(3):141-149. 